\title{
APLICAÇÃO DO PROCESSO DE ENFERMAGEM BASEADO NA TEORIA DE OREM: ESTUDO DE CASO COM UMA ADOLESCENTE GRÁVIDA
}

\author{
Gilson de Vasconcelos Torres* \\ Rejane Marie Barbosa Davim** \\ Maria Miriam Lima da Nóbrega***
}

TORRES, G.de V.; DAVIM, R.M.B.; NÓBREGA, M.M.L.da. Aplicação do processo de enfermagem baseado na teoria de OREM: estudo de caso com uma adolescente grávida. Rev.latino-am.enfermagem, Ribeirão Preto, v. 7, n. 2, p. 47-53, abril 1999.

Estudo do tipo descritivo com abordagem qualitativa, objetivando a aplicação do processo de enfermagem baseado na Teoria do Autocuidado de Orem, através de um estudo de caso em uma adolescente grávida, buscando identificar os diagnósticos de enfermagem na referida cliente, baseado nos diagnósticos de enfermagem da NANDA. Diante dos resultados obtidos identificouse três diagnósticos: adaptação prejudicada, distúrbio do padrão do sono e processo familiar alterado. Destacou-se a aplicabilidade do processo de enfermagem baseado em Orem e a importância dos diagnósticos de enfermagem na prestação dos cuidados de enfermagem à cliente.

UNITERMOS: diagnóstico de enfermagem, adolescente grávida, teoria do autocuidado

\section{INTRODUÇÃO}

A adolescência apresenta-se como um período muito importante na vida do indivíduo, constituindo etapa decisiva e um processo de transição conflitante, a qual está exposta a muitos agravos, como por exemplo, as doenças sexualmente transmissíveis (DST), o consumo de drogas, do álcool, e uma atividade sexual precoce. Tudo isto pode gerar, de certa forma, crises de alto grau de ansiedade, tornando os adolescentes vulneráveis, acarretando também, a debilidade física e mental dos mesmos, bem como, a maternidade e paternidade precoces.

Um dos grandes conflitos dos adolescentes, é a busca de uma noção coerente de identidade, onde os mesmos não se definem, buscando apoio em grupos ou em qualquer coisa que os identifique, como o andar, o vestir, o falar gíria, fazendo com que os mesmos sintamse seguros e que tudo isto seja comum em sua idade.

Segundo BEZERRA et al. (1988), a adolescente brasileira é do ponto de vista biológico e psicológico semelhante às adolescentes de outros países, só que a realidade sócio-econômica-cultural na qual se insere é diversa. Como the foi dado o direito ao exercício da sexualidade por uma revolução sexual, não foi observada entretanto, a forma de lhe assegurar tais direitos, fazendo com que essas jovens por não terem atingido a completa maturidade, tenham maior possibilidade de início precoce de atividades sexuais, o que implica em risco de DST e gravidez indesejada para as que não se previnem, seja por imprudência, ou mesmo por desconhecimento dos meios como evitá-los.

Falando-se sobre a gravidez na adolescência, SILVA \& SARMENTO (1988), comentam que nestas três últimas décadas, este assunto tem sido objeto de discussão tanto nos meios acadêmicos como nos meios de comunicação de massa. Sua incidência tem aumentado muito segundo as estatísticas de vários países, tornandose uma situação muito preocupante, pois a maternidade nessa faixa etária é considerada como problemática do ponto de vista biopsicossocial.

Como a gravidez na adolescência ocorre inesperadamente, isto vai acarretar uma série de episódios negativos interferindo no desenvolvimento da jovem,

\footnotetext{
* Professor Assistente do Departamento de Enfermagem da UFRN - Mestre em Enfermagem de Saúde Pública/UFPB. Doutorando em Enfermagem Fundamental da Escola de Enfermagem de Ribeirão Preto da Universidade de São Paulo

** Professora Adjunto do Departamento de Enfermagem da UFRN - Mestre em Enfermagem de Saúde Pública da UFPB

*** Professora Mestre do Mestrado de Enfermagem de Saúde Pública da UFPB - Doutoranda em Enfermagem da PUC/USP Orientadora do trabalho
} 
como por exemplo, rejeição familiar, restrições sociais e econômicas. Sendo assim, a adolescente entra em crise, a qual é determinada por um fator criticamente duplo: a crise da adolescência, somando-se à crise da gravidez.

Tem-se observado também, que a atividade sexual está ocorrendo cada vez mais cedo em todo o mundo, fazendo com que as chances de gravidez precoce ocorram com maior intensidade. Para RODRIGUES et al. (1993), essa atividade sexual na maioria das adolescentes inicia-se aproximadamente dois anos após a menarca, onde $20 \%$ delas tornam-se grávidas. No Brasil, segundo os mesmos autores, 5 em cada 10 adolescentes tornam-se sexualmente ativas antes mesmo dos 20 anos de idade.

Dessa forma, LUZ (1989), considerando a gravidez na adolescência como um problema de saúde pública em nosso meio e de difícil solução, acredita que seja necessária a implementação de programas de atenção à saúde dessas jovens, chamando a atenção para o poder público a fim de estimular e subsidiar programas de saúde ao nível preventivo para esse grupo etário.

Diante de todas estas considerações, resolvemos desenvolver um estudo, aplicando o processo de enfermagem baseado na teoria do autocuidado de Dorothea Orem com uma adolescente grávida solteira, buscando detectar os diagnósticos de enfermagem, utilizando-se, por conseguinte, os diagnósticos de enfermagem propostos na Taxonomia dos Diagnósticos de Enfermagem pela North American Nursing Diagnosis Association da NANDA. Pretendemos, também, estabelecer objetivos e traçar um plano assistencial no sentido de melhorar as condições de saúde e bem-estar que essa jovem tenha para cuidar de si mesma, tendo-se em vista, o respeito pelas sugestões e decisões da pesquisada.

Entretanto, na literatura, encontramos poucos trabalhos sobre diagnósticos de enfermagem relacionados a adolescentes grávidas. Porém, dentre as publicações encontradas, podemos citar o trabalho, Maternidade na adolescência: escolha ou fatalidade? realizado por GARCIA (1992), o qual discute a adequação dos diagnósticos de enfermagem à problemática social das gestantes adolescentes solteiras, captada a partir das representações dessas gestantes sobre o seu vivido humano social. Outro trabalho realizado por GARCIA (1996) sob o título, Cuidando de adolescentes grávidas solteiras, a autora utilizou a teoria de Imogene King e demonstrou a inter-relação e interdependência dos aspectos psicobiológicos, psicoemocionais e psicossociais.

Acreditamos, portanto, na importância dos diagnósticos de enfermagem, e esperamos que os enfermeiros passem a trabalhar com mais ênfase no campo da adolescência, adquirindo, assim, habilidades no uso dos diagnósticos de enfermagem nessa área.

\section{2- REVISÃO DA LITERATURA}

\section{1 - Teoria do autocuidado de Dorothea Orem}

A teoria do autocuidado de Orem, engloba o autocuidado, a atividade de autocuidado e a exigência terapêutica de autocuidado. O autocuidado, é a prática de atividades iniciadas e executadas pelos indivíduos em seu próprio benefício para a manutenção da vida e do bem-estar. A atividade de autocuidado, constitui uma habilidade para engajar-se em autocuidado. A exigência terapêutica de autocuidado, constitui a totalidade de ações de autocuidado, através do uso de métodos válidos e conjuntos relacionados de operações e ações (FOSTER \& JANSSENS, 1993).

Para OREM (1980), o autocuidado é a prática de atividades que o indivíduo inicia e executa em seu próprio benefício, na manutenção da vida, da saúde e do bemestar. Tem como propósito, as ações, que, seguindo um modelo, contribui de maneira específica, na integridade, nas funções e no desenvolvimento humano. Esses propósitos são expressos através de ações denominadas requisitos de autocuidado.

São três os requisitos de autocuidado ou exigências, apresentados por Orem: universais, de desenvolvimento e de desvio de saúde.

Os universais estão associados a processos de vida e à manutenção da integridade da estrutura e funcionamento humanos. Eles são comuns a todos os seres humanos durante todos os estágios do ciclo vital, como por exemplo, as atividades do cotidiano.

Os requisitos de desenvolvimento são as expressões especializadas de requisitos universais que foram particularizados por processos de desenvolvimento, associados a algum evento; por exemplo, a adaptação a um novo trabalho ou adaptação a mudanças físicas.

O de desvio de saúde é exigido em condições de doença, ferimento ou moléstia, ou pode ser conseqüência de medidas médicas exigidas para diagnosticar e corrigir uma condição.

Dessa forma, POLIT \& HUNGLER (1995), afirmam que a capacidade que o indivíduo tem para cuidar de si mesmo, é chamada de intervenção de autocuidado, e a capacidade de cuidar dos outros é chamada de intervenção de cuidados dependentes. Sendo assim, no modelo de Orem, a meta é ajudar as pessoas a satisfazerem suas próprias exigências terapêuticas de autocuidado.

Portanto, a teoria do autocuidado de Orem segundo LUCE et al. (1990), tem como premissa básica, 
a crença de que o ser humano tem habilidades próprias para promover o cuidado de si mesmo, e que pode se beneficiar com o cuidado da equipe de enfermagem quando apresentar incapacidade de autocuidado ocasionado pela falta de saúde.

\section{2. - A teoria de déficit de autocuidado}

Segundo FOSTER \& JANSSENS (1993), a teoria de déficit de autocuidado constitui a essência da teoria de Orem, quando a enfermagem passa a ser uma exigência a partir das necessidades de um adulto, e quando o mesmo acha-se incapacitado ou limitado para prover autocuidado contínuo e eficaz. Esses cuidados podem ser oferecidos pela enfermagem quando

[...] as habilidades para cuidar sejam menores do que as exigidas para satisfazer uma exigência conhecida de autocuidado[ ...] ou habilidades de autocuidados ou de cuidados dependentes excedam ou igualem às exigidas para satisfazer a demanda atual de autocuidado, embora uma relação futura de deficiência possa ser prevista devido as diminuições previsíveis de habilidades de cuidado (FOSTER \& JANSSENS, 1993, p.92).

Orem identificou cinco métodos de ajuda, segundo os autores anteriormente citados: 1) agir ou fazer para o outro; 2) guiar o outro; 3 ) apoiar o outro (física ou psicologicamente); 4) proporcionar um ambiente que promova o desenvolvimento pessoal, quanto a tornar-se capaz de satisfazer demandas futuras ou atuais de ação; e 5) ensinar o outro.

\section{3 - A teoria de sistemas de enfermagem}

O sistema de enfermagem planejado pelo profissional, segundo FOSTER \& JANSSENS (1993), está baseado nas necessidades de autocuidado e na capacidade do paciente para a execução de atividades de autocuidado. Para satisfazer os requisitos de autocuidado do indivíduo, Orem identificou três classificações de sistemas de enfermagem que são os seguintes: o sistema totalmente compensatório, o sistema parcialmente compensatório e o sistema de apoio-educação.

$\mathrm{O}$ sistema de enfermagem totalmente compensatório é representado pelo indivíduo incapaz de empenhar-se nas ações de autocuidado. O enfermeiro, através de suas ações, vai atuar na ação limitada do paciente conseguindo o autocuidado do mesmo, compensando sua incapacidade para a atividade de autocuidado através do apoio e da proteção ao paciente.

$\mathrm{O}$ sistema de enfermagem parcialmente compensatório está representado por uma situação em que, tanto o enfermeiro, quanto o paciente, executam medidas ou outras ações de cuidado que envolvem tarefas de manipulação ou de locomoção. Através de sua ação, o enfermeiro efetiva algumas medidas de autocuidado pelo paciente, compensa suas limitações de autocuidado atendendo o paciente conforme o exigido. $O$ paciente age realizando algumas medidas de autocuidado, regula suas atividades e aceita atendimento e auxílio do enfermeiro.

$\mathrm{O}$ sistema de enfermagem de apoio-educação ocorre quando o indivíduo consegue executar, ou pode e deve aprender a executar medidas de autocuidado terapêutico, regula o exercício e desenvolvimento de suas atividades de autocuidado, e o enfermeiro vai promover esse indivíduo a um agente capaz de se autocuidar.

\section{4 - O processo de enfermagem de OREM}

O processo de enfermagem de Orem segundo FOSTER \& JANSSENS (1993, p.98) "é um método de determinação das deficiências de autocuidado e a posterior definição dos papéis da pessoa ou enfermeiro para satisfazer as exigências de autocuidado".

O processo de enfermagem proposto por OREM (1980) compreende os seguintes passos, segundo os mesmos autores:

Passo 1 - fase de diagnóstico e prescrição, que determina as necessidades ou não de cuidados de enfermagem. $\mathrm{O}$ enfermeiro realiza a coleta de dados do indivíduo. Os dados específicos são reunidos nas áreas das necessidades de autocuidado, de desenvolvimento e de desvio de saúde do indivíduo, bem como, o seu inter-relacionamento. São também coletados dados acerca dos conhecimentos, habilidades, motivação e orientação da pessoa.

Passo 2 - é a fase do planejamento dos sistemas de enfermagem, bem como do planejamento da execução dos atos de enfermagem. O enfermeiro cria um sistema que seja totalmente compensatório, parcialmente compensatório ou de apoio-educação. As duas ações envolvidas no planejamento dos sistemas de enfermagem seriam: a) a realização de uma boa organização dos componentes das exigências terapêuticas de autocuidado dos pacientes; b) a seleção da combinação de maneiras de auxílio que sejam, ao mesmo tempo, efetivas e eficientes na tarefa de compensar ou sobrepujar os déficits de autocuidado dos pacientes. Com a utilização do modelo de Orem, as metas são compatíveis com o diagnóstico de enfermagem, capacitando o paciente a tornar-se um verdadeiro agente de autocuidados.

Passo 3 - inclui a produção e execução do sistema de enfermagem, onde o enfermeiro pode prestar auxílio ao indivíduo (ou família) no que se refere ao autocuidado, de modo a alcançar resultados identificados e descritos de saúde. O passo 3 inclui a evolução, onde, juntos, paciente e enfermeiro, realizam a avaliação. A evolução é um processo contínuo, e é fundamental que o enfermeiro e o paciente avaliem quaisquer modificações nos dados que afetariam o déficit de autocuidado, o agente de autocuidado e o sistema de enfermagem. 


\section{3 - PROCEDIMENTOS METODOLÓGICOS}

Este é um estudo descritivo com uma abordagem qualitativa, do tipo estudo de caso, objetivando identificar os diagnóstico de enfermagem em uma adolescente grávida, utilizando-se para isto, a Uniformização da Linguagem dos Diagnósticos de Enfermagem da NANDA: sistematização das propostas do II SNDE, conforme apresentado e descrito por NÓBREGA \& GARCIA (1994), e o processo de enfermagem baseado na teoria do autocuidado de Dorothea Orem.

O mesmo foi realizado no ambulatório de uma maternidade-escola, na cidade de Natal, RN. A escolha dessa instituição deveu-se ao fato de ser um campo de ensino universitário especializado no atendimento à adolescentes, por ser um hospital de referência do Estado do Rio Grande do Norte e por apresentar o principal campo de prática no acompanhamento a alunos de um dos pesquisadores do trabalho na disciplina de enfermagem obstétrica, na graduação.

A amostra foi constituída por uma adolescente grávida solteira, inscrita no programa de pré-natal da referida instituição, e selecionada através de um processo aleatório simples. A coleta de dados foi desenvolvida, seguindo-se um roteiro de entrevista (Anexo) fundamentado e adaptado à teoria do autocuidado de Orem.

A coleta de dados procedeu-se na residência da cliente por um dos autores do trabalho, após esclarecimentos à mesma quanto a finalidade da pesquisa e obtenção de sua anuência. Foram coletados dados a partir da entrevista, da observação e do exame físico da adolescente. Para a operacionalização do processo de enfermagem baseado no autocuidado de Orem, foram realizadas oito visitas domiciliares à adolescente durante um mês, sendo duas visitas por semana. Esta operacionalização seguiu os seguintes passos do processo: $1^{\mathrm{o}}$ passo - realização do levantamento de dados através da entrevista;

$2^{\circ}$ passo - planejamento das ações de enfermagem através do sistema de apoio-educação;

$3^{\circ}$ passo - execução e avaliação das ações de enfermagem no sistema escolhido.

Após concluída a fase de coleta de dados, foi iniciado o trabalho de análise das informações coletadas. Para se chegar aos diagnósticos, teve-se como base, as características definidoras e os fatores relacionados determinados pela classificação da NANDA com adaptação ao nosso meio conforme NÓBREGA \& GARCIA (1994), como também o conhecimento e a experiência dos autores na sistematização da assistência de enfermagem.

Previamente à coleta, foi solicitado a assinatura de uma carta de consentimento da mãe da adolescente autorizando sua participação no estudo, obedecendo, assim, os preceitos éticos e legais para menores de 18 anos. Tornamos evidente à participante do estudo, que lhe seria assegurado o anonimato, resguardando-lhe o direito, inclusive, de não concluir a entrevista, se assim o desejasse.

\section{1 - Estudo de caso correspondente ao binômio adolescente grávida solteira/família}

A cliente T.S.F. reside com a mãe, tem 17 anos de idade, com 1,74 $\mathrm{m}$ de altura e $65 \mathrm{Kg}$ de peso corporal, grávida de 7 meses de seu primeiro filho, solteira, gravidez não planejada, católica, cursando o segundo ano do segundo grau. Refere não estar dormindo bem à noite, acordando várias vezes justificando pela mudança nos hábitos de dormir após a gravidez (antes dormia em decúbito ventral, agora dorme em decúbito lateral). Está preparando o enxoval do bebê, mas não com muita expectativa porque seu companheiro não dá nenhuma ajuda financeira. Antes da gravidez estudava em colégio particular, teve que sair porque seu pai deixou de pagar seus estudos quando soube da gravidez, deixando até de falar com a mesma, e que a mudança de colégio não foi boa "... porque onde eu estudava o ensino era bom ...". Quando descobriu que estava grávida, diz que, “... chorei muito, mas de raiva ... " porém com o evoluir da gravidez foi se adaptando à sua nova situação. $\mathrm{O}$ companheiro reconheceu sua gravidez aceitando a paternidade, freqüentava sua residência, porém agora não comparece mais, devido a incompatibilidade do mesmo com a mãe da jovem, a qual relaciona essa situação pelo seu estado de gravidez. Quanto ao ambiente familiar em termos emocionais, refere “... que está insustentável, minha mãe não está feliz com esta nova situação ..." Quanto às suas expectativas, não sabe responder, e que “ ... sonhos são só sonhos, e talvez ainda não esteja no tempo de saber. Minha cabeça está a mil, em parafuso. Acho que no momento estou mais para o colégio do que para a gravidez. Não engravidem crianças, pois amar não vale a pena, nada é eterno".

\section{4 - RESULTADOS}

Os resultados encontrados nos levaram a identificar e nomear os diagnósticos de enfermagem referentes aos padrões de resposta humana ESCOLHER (adaptação prejudicada), MOVER (distúrbio do padrão do sono) e RELACIONAR (processo familiar alterado), descritos a seguir no Quadro 1. 
QUADRO 1 - Plano de enfermagem baseado na teoria do autocuidado de Orem para os Padrões de Resposta Humana ESCOLHER, MOVER e RELACIONAR - Natal/RN - 1996

\begin{tabular}{|c|c|c|c|}
\hline Diagnóstico de Enfermagem & Plano & Intervenção & Evolução \\
\hline $\begin{array}{l}\text { 1- Adaptação prejudicada } \\
\text { relacionado ao sistema de } \\
\text { suporte inadequado }\end{array}$ & $\begin{array}{l}\text { a) Meta: } \\
\text { - Levar a cliente a adaptar-se } \\
\text { à sua nova situação } \\
\text { b) Objetivos: } \\
\text { - Dar condições a uma melhor } \\
\text { adaptação da gravidez da } \\
\text { cliente e a sua mudança de } \\
\text { colégio } \\
\text { c) Sistema de enfermagem: } \\
\text { - De apoio-educação } \\
\text { d) Método: } \\
\text { - Orientação e apoio }\end{array}$ & $\begin{array}{l}\text { - Orientar a cliente sobre a } \\
\text { aceitação de sua gravidez } \\
\text { através do diálogo, e dar apoio } \\
\text { quanto a mudança no seu } \\
\text { padrão de vida com uma } \\
\text { melhor aceitação para o novo } \\
\text { colégio }\end{array}$ & $\begin{array}{l}\text { - A cliente vem aceitando sua } \\
\text { gravidez; já freqüenta as aulas } \\
\text { no novo colégio, relaciona-se } \\
\text { com novos colegas e pensa } \\
\text { concluir seu curso secundário }\end{array}$ \\
\hline $\begin{array}{l}\text { 2- Distúrbio do padrão do sono } \\
\text { relacionado a alterações } \\
\text { sensoriais internas }\end{array}$ & $\begin{array}{l}\text { a) Meta: } \\
\text { - Promover sono e repouso } \\
\text { adequado às suas } \\
\text { necessidades fisiológicas } \\
\text { b) Objetivo: } \\
\text { - Tentar adaptar a cliente a } \\
\text { novas posições para o sono e } \\
\text { repouso } \\
\text { c) Sistema de enfermagem: } \\
\text { - De apoio-educação } \\
\text { d) Método: } \\
\text { - Orientação e ensino }\end{array}$ & $\begin{array}{l}\text { - Orientar e demonstrar a } \\
\text { cliente novas técnicas para } \\
\text { mudança postural: } \\
\text {. decúbito ventral com apoio de } \\
\text { travesseiros sob o tórax } \\
\text {. decúbito lateral apoiando o } \\
\text { abdomen sobre os travesseiros } \\
\text {. colocar travesseiros sob as } \\
\text { pernas quando em decúbito } \\
\text { dorsal e posição de semi-fowler }\end{array}$ & $\begin{array}{l}\text { - A cliente já consegue dormir } \\
\text { e repousar com o apoio de } \\
\text { travesseiros como foi ensinado }\end{array}$ \\
\hline $\begin{array}{l}\text { 3- Processo familiar alterado } \\
\text { relacionado à situação de } \\
\text { transição }\end{array}$ & $\begin{array}{l}\text { a) Meta: } \\
\text { - Reduzir o desequilíbrio e } \\
\text { desajuste familiar } \\
\text { b) Objetivo: } \\
\text { - Melhorar o relacionamento } \\
\text { e o convívio familiar } \\
\text { c) Sistema de enfermagem: } \\
\text { - De apoio-educação } \\
\text { d) Método: } \\
\text { - Orientação e apoio }\end{array}$ & $\begin{array}{l}\text { - Orientar a cliente e seus } \\
\text { familiares sobre a situação } \\
\text { transitória que estão passando } \\
\text { e apoiar os mesmos nas } \\
\text { discussões dos problemas, } \\
\text { através do diálogo }\end{array}$ & $\begin{array}{l}\text { - A cliente refere que os seus } \\
\text { pais já estão aceitando a } \\
\text { gravidez e a presença do } \\
\text { companheiro no convívio } \\
\text { familiar (só a mãe), a qual } \\
\text { demonstra interesse em sua } \\
\text { gravidez e o companheiro } \\
\text { começou a ajudar na compra do } \\
\text { enxoval do bebê }\end{array}$ \\
\hline
\end{tabular}

\section{5- CONSIDERAÇÕES FINAIS}

É possível considerar que o uso da teoria do autocuidado de Dorothea Orem é um instrumento válido, o qual nos ajudou a promover uma comunicação mais objetiva entre pesquisador e pesquisada, adequando-se de certa forma ao planejamento da assistência de enfermagem, à problemática dessa adolescente. Considera-se também, que o processo de enfermagem baseado em Orem, nos deu subsídios para a aplicação sistemática dessa assistência, fazendo-se mudanças necessárias ao plano de cuidados mesma.

Dessa forma, diante dos resultados obtidos no estudo de caso, os diagnósticos de enfermagem encontrados foram: adaptação prejudicada, distúrbio do padrão do sono e processo familiar alterado. No padrão de resposta humana ESCOLHER, identificou-se o diagnóstico adaptação prejudicada, que se traduz pelo descontentamento da jovem em estar grávida e por sair do colégio privado, devido a revolta do pai sobre sua gestação, vindo assim, conturbar suas ilusões e fantasias de adolescente. Na cliente deste estudo com o diagnóstico em questão, foram identificadas reações variáveis que envolveram a demonstração da incapacidade para a resolução de seus problemas atuais, incapacidade de alcançar e desempenhar papéis desejáveis, mudanças na sua vida social, expressos sob a forma de raiva e hostilidade, isolamento, medo, decepção e negação.

No padrão de resposta humana MOVER, podese identificar o diagnóstico distúrbio do padrão do sono, 
caracterizado pela mudança dos hábitos de dormir da cliente, durante sua gravidez.

No padrão de resposta humana RELACIONAR, identificou-se o diagnóstico processo familiar alterado, definido pela interação familiar prejudicada, tanto com os pais, quanto com o companheiro.

Embora este trabalho represente um estudo preliminar no levantamento de possíveis diagnósticos de enfermagem identificados em uma adolescente grávida solteira, o mesmo nos permitiu entender que o diagnóstico de enfermagem não pode ser uma fase isolada de todo o processo assistencial, mas, que deve ser utilizado com o objetivo de direcionar a ação de enfermagem.

A incorporação dos diagnósticos de enfermagem propostos pela NANDA, nos permitiu confirmar a concepção que os autores do trabalho já possuíam, que a assistência a uma adolescente grávida não pode ser voltada somente para o aspecto biológico, mas também, para o social e o psicológico. Cabe ressaltar, ainda, a necessidade de outros estudos nessa área junto a adolescentes grávidas, em especial solteiras, para a definição de um perfil diagnóstico, a fim de subsidiar a assistência de enfermagem sistematizada e fundamentada cientificamente.

\section{APPLICATION OF THE NURSING PROCESS BASED IN OREM'S THEORY: A CASE STUDY WITH A PREGNANT ADOLESCENT}

This is a descriptive study with a qualitative approach that aimed at applying the nursing process based in Orem SelfCare Theory, through a case study with a pregnant adolescent in order to identify the nursing diagnosis in the above mentioned clients, based on NANDA'S nursing diagnoses. Results obtained identified three nursing diagnoses: prejudiced adaptation, sleep disturb and familiar change process. The application of the nursing process based in Orem and the importance of the diagnosis identified for clients nursing care were evidenced.

\section{APLICACIÓN DE LO PROCESO DE ENFERMERÍA BASADO EN LA TEORÍA DE OREM: ESTUDIO DE CASO EN UNA ADOLESCENTE EMBARAZADA}

Estudio de tipo descriptivo con un enfoque cualitativo, cuyo objetivo fue aplicar el proceso de enfermería utilizando la Teoría del Autocuidado de OREM, a través de un estudio de caso en una adolescente embarazada, buscando identificar los diagnósticos de la cliente, en base a los diagnósticos de la NANDA. De acuerdo con los resultados encontrados, se identificaron tres diagnósticos: adaptación perjudicada, disturbio del sueño y alteración del proceso familiar, fue relevante la aplicación del proceso de enfermería de Orem y la importancia de los diagnósticos de enfermería para dar los cuidados de enfermería a la cliente.

TÉRMINOS CLAVES: diagnóstico de enfermería, adolescente embarazada, teoría del autocuidado

\section{ANEXO \\ ROTEIRO DE ANAMNESE}

1- Fatores pessoais e condicionantes básicos:

1.1. Identificação:

Nome:

Sexo:

Estado civil:

Escolaridade:

Altura:

Peso:

Idade:

Filhos:

Profissão:

Religião:

$\mathrm{N}^{\circ}$ gestações:

$\mathrm{N}^{\mathrm{o}}$ aborto:

2- Requisitos de desenvolvimento:

2.1. Antecedentes pessoais

- Doenças da infância

- Sexualidade

- Menarca

- Gravidez

- Relacionamento familiar

3- Requisitos universais para o autocuidado:
3.1. Exame físico

- Higiene corpo

- Tórax

- Abdome

- Sistema urinário

- Sistema intestinal

- Membros inferiores

- Sistema neurológico

- Nível de consciência

- Sistema reprodutor: número de filhos, uso de contraceptivos - Alimentação: Principais alimentos que ingere, dieta e número de refeições diárias

- Atividades e repouso

- Atividades, lazer, repouso

- Hábitos e vícios

4- Desvio de saúde:

4.1. Queixas atuais:

Percepção sobre a sua gravidez atual

Conhecimento sobre a gravidez e suas intercorrências

Orientações recebidas e exames realizados 


\section{REFERÊNCIAS BIBLIOGRÁFICAS}

01. BEZERRA, M.L. et al. Estudo retrospectivo sobre adolescentes grávidas atendidas no Instituto Materno-Intantil de Pernambuco, no biênio 1984/ 1985. Rev.Bras.Ginecol.Obstet., Rio de Janeiro, v. 10, n. 10, p. 223-226, 1988.

02. FOSTER, P.C.; JANSSENS, N.P. D.E.O. In: GEORGE, J.B. et al. Teorias de Enfermagem. Porto Alegre: Artes Médicas, 1993. Cap. 7, p. 90-107.

03. GARCIA, T.R. Maternidade na adolescência: escolha ou fatalidade? Rev.Bras.Enfermagem, Brasília, v. 45, n. 1, p. 44-53, jan./mar. 1992.

04. GARCIA, T.R. Cuidando de adolescentes grávidas solteiras. Ribeirão Preto, 1996. 256p. Tese (Doutorado) - Escola de Enfermagem de Ribeirão Preto, Universidade de São Paulo.

05. LUCE, M. et al. O preparo para o autocuidado do cliente diabético e família. Rev. Bras. Enfermagem, Brasília, v. 1, n. 1/2/3/4, p. 36-49, jan./dez. 1990.
06. LUZ, A.M.H. Proposta de programa de assistência a adolescentes gestantes (com base em estudo de mães adolescentes e adultas). Rev.Gauch.Enfermagem, Porto Alegre, v. 10, n. 2, p. 69-79, jul. 1989.

07. NÓBREGA, M.M.L.da; GARCIA, T.R. Uniformização da linguagem dos diagnósticos de enfermagem da NANDA: sistematização das propostas do II SNDE. João Pessoa: A União, CNRDE/GIDE, 1994. 138p.

08. OREM, D.E. Nursing: concepts of practice. 2. ed. New York: McGrau-Hill, 1980. Ch.3, p. 35-54: Nursing and self-care.

09. POLIT, D.F.; HUNGLER, B.P. Fundamentos de pesquisa em enfermagem. 3. ed. Porto Alegre: Artes Médicas, 1995. Cap. 8, p. 163-198: Métodos de coleta de dados.

10. RODRIGUES, A.P. et al. Gravidez na adolescência. Femina, Rio de Janeiro, v. 21, n. 3, p. 199-223, mar. 1993.

11. SILVA, J.L.P.; SARMENTO, R.C. Gravidez. In: COMISSÃO DE SAÚDE DO ADOLESCENTE. Adolescência e saúde. São Paulo: Paris, 1988. Cap. 11, p. 131-142. 